\title{
Investigation of an angular spectrum approach for pulsed ultrasound fields
}

\author{
Du, Yigang; Jensen, Henrik ; Jensen, Jørgen Arendt
}

Published in:

Ultrasonics

Link to article, DOI:

10. 1016/j.ultras .2013.0 2.011

Publication date:

2013

Document Version

Early version, also known as pre-print

Link back to DTU Orbit

Citation (APA):

Du, Y., Jensen, H., \& Jensen, J. A. (2013). Investigation of an angular spectrum approach for pulsed ultrasound fields. Ultrasonics, 53(11), 1185-1191. https://doi.org/10. 1016/j.ultras .2013.0 2.011

\section{General rights}

Copyright and moral rights for the publications made accessible in the public portal are retained by the authors and/or other copyright owners and it is a condition of accessing publications that users recognise and abide by the legal requirements associated with these rights.

- Users may download and print one copy of any publication from the public portal for the purpose of private study or research.

- You may not further distribute the material or use it for any profit-making activity or commercial gain

- You may freely distribute the URL identifying the publication in the public portal

If you believe that this document breaches copyright please contact us providing details, and we will remove access to the work immediately and investigate your claim. 


\title{
Investigation of an angular spectrum approach for pulsed ultrasound fields
}

\author{
Yigang $\mathrm{Du}^{\mathrm{a}, \mathrm{b}, *}$, Henrik Jensen $^{\mathrm{b}}$, Jørgen Arendt Jensen ${ }^{\mathrm{a}}$ \\ ${ }^{a}$ Center for Fast Ultrasound Imaging, Department of Electrical Engineering, Technical University of Denmark, DK-2800 Kgs. Lyngby, Denmark \\ ${ }^{\mathrm{b}}$ BK Medical Aps, DK-2730 Herlev, Denmark
}

\section{A R T I C L E I N F O}

\section{Article history:}

Received 29 May 2011

Received in revised form 31 January 2013

Accepted 22 February 2013

Available online $\mathrm{xxxx}$

\section{Keywords:}

Angular spectrum approach

Pulsed ultrasound field

Field II

Ambient sound speed approximation

\begin{abstract}
A B S T R A C T
An Angular Spectrum Approach (ASA) is formulated and employed to simulate linear pulsed ultrasound fields for high bandwidth signals. A geometrically focused piston transducer is used as the acoustic source. Signals are cross-correlated to find the true sound speed during the measurement to make the simulated and measured pulses in phase for comparisons. The calculated sound speed in the measurement is varied between $1487.45 \mathrm{~m} / \mathrm{s}$ and $1487.75 \mathrm{~m} / \mathrm{s}$ by using different initial values in the ASA simulation. Results from the pulsed ASA simulations using both Field II simulated and hydrophone measured acoustic sources are compared to the Field II simulated and hydrophone measured pulses, respectively. The total relative root mean square (RMS) errors of the pulsed ASA are investigated by using different time-point, zero-padding factors, spatial sampling interval and temporal sampling frequency in the simulation. Optimal parameters for the ASA are found in the simulation. The RMS error of the ASA simulation is reduced from $10.9 \%$ to $2.4 \%$ for the optimal parameters when comparing to Field II simulations. The comparison between the ASA calculated and measured pulses are illustrated and the corresponding RMS error is $25.4 \%$.
\end{abstract}

() 2013 Elsevier B.V. All rights reserved.

\section{Introduction}

Monochromatic acoustic fields can be simulated using the Angular Spectrum Approach (ASA) [1-3]. Here the acoustic pressure is decomposed into a summation of an infinite numbers of plane waves in $k$-space. An arbitrary shaped two-dimensional plane in space is assumed as the acoustic source. After propagation, the pressure at any plane parallel to the source plane can be expressed as the multiplication between the source pressure and a propagating distance dependent propagator in the spatial Fourier domain. Mathematically, it originates from Fourier optics [4,5]. However, it has been widely used in ultrasound simulations due to its rapid computation time [6,7]. Simulations of spherically focused ultrasound phased arrays using the ASA have been shown by Zeng and McGough [8]. Wu and Stepinski [9] have applied the ASA to concave ultrasound transducers. Moreover, the ASA has been applied to simulate non-linear ultrasound propagation. This was firstly introduced by Alais and Hennion [10]. Landsberger and Hamilton [11] have studied the second harmonic generation of sound beams in immersed elastic solids. An application of angular spectrum decomposition of the second harmonic ultrasound to tissue harmonic imaging was given by Xiang and Hamilton [12]. A validation of the ASA for simulating fundamental and second harmonic fields with measured results have been published in the pre-

\footnotetext{
* Corresponding author.

E-mail address: yigang1982@gmail.com (Y. Du).
}

vious work [13]. These studies of linear and non-linear pressure fields simulation using the ASA are usually restricted to monochromatic fields.

For realistic prediction of ultrasound imaging, pulsed ultrasound fields should be simulated. Several studies have been made for the transient pressure field. Leeman and Healey $[14,15]$ have proposed a technique based on the ASA for simulating transient fields, but the measurement was restricted by the quality of the hydrophone in [14] and no measured result was shown for the validation in [15]. Wu et al. [16] presented their results compared to a measurement in water and the ASA employed the pulsed normal velocity as its source, which was calculated numerically. Some studies $[17,18]$ focused on non-linear ultrasound also formulated the ASA for simulating the linear pulsed pressure field.

In this paper results calculated by the ASA using both the simulated and measured sources are presented. This makes the comparison for a pulse at a specific position possible. For the simulated ASA source, two acoustic planes with a certain distance parallel to the transducer surface are simulated by the Field II program $[19,20]$. One of the planes is assumed as the simulated virtual source plane of the ASA. The pressure on the second plane is calculated by the pulsed ASA based on the simulated source and distance between two planes. Finally, the pulses obtained from the ASA on the second plane are compared to the results obtained from Field II. For the measured ASA source, two acoustic planes are measured by a robust hydrophone in water. The first measured plane is used as the source for the ASA calculation. The results obtained at 
the second plane by the ASA are compared to the measurements. For the ASA with measured source, an approach to finding the ambient acoustic propagation speed in the measurement is presented.

\section{Theory}

In this section, the equations for the pulsed ASA are presented. The angular spectrum of the acoustic pressure is introduced. The angular spectrum solutions are given both for monochromatic and pulsed fields. The implemented equations are presented in discrete form at the end of this section.

\subsection{Calculation of monochromatic ultrasound fields}

For a monochromatic ultrasound field, the two-dimensional spatial Fourier transform of a pressure field at a given plane is called the angular spectrum, denoted by $\widehat{P}\left(k_{x}, k_{y}, z\right)$, where $k_{x}$ and $k_{y}$ are transverse wave numbers along $x$ and $y$ directions in the spatial frequency domain, and $z$ represents the position of the given plane in the $z$ direction. The relation between the two angular spectra $\widehat{P}_{0}$ and $\widehat{P}_{1}$ at different planes, can be expressed by $[4,5]$

$\widehat{P}_{1}\left(k_{x}, k_{y}, z_{1}\right)=\widehat{P}_{0}\left(k_{x}, k_{y}, z_{0}\right) H_{p}\left(k_{x}, k_{y}\right)$,

where

$H_{p}\left(k_{x}, k_{y}\right)= \begin{cases}e^{-j\left(z_{1}-z_{0}\right) \sqrt{k^{2}-k_{x}^{2}-k_{y}^{2}}}, & k^{2}>k_{x}^{2}+k_{y}^{2} \\ e^{-\left(z_{1}-z_{0}\right) \sqrt{k_{x}^{2}+k_{y}^{2}-k^{2}}}, & k^{2} \leqslant k_{x}^{2}+k_{y}^{2} .\end{cases}$

In (1), $\widehat{P}_{0}\left(k_{x}, k_{y}, z_{0}\right)$ is usually considered as the acoustic source to predict $\widehat{P}_{1}\left(k_{x}, k_{y}, z_{1}\right)$, which is the angular spectrum at the $z_{1}$ plane. This is the conventional ASA used to simulate the propagation of monochromatic acoustic fields, and it is assumed that the temporal frequency $f$ is a constant and not a function of $\widehat{P}$ in the equation.

\subsection{Calculation of pulsed ultrasound fields}

For a high bandwidth signal, the angular spectrum corresponding to each temporal frequency in the temporal Fourier domain, is written as

$\widehat{P}\left(k_{x}, k_{y}, z, f\right)=\iint_{-\infty}^{\infty} P(x, y, z, f) e^{-j\left(k_{x} x+k_{y} y\right)} d x d y$,

where $P(x, y, z, f)$ is the temporal Fourier transform of the pressure of ultrasound pulses and defined by

$P(x, y, z, f)=\int_{-\infty}^{\infty} p(x, y, z, t) e^{-j 2 \pi f t} d t$,

where $p(x, y, z, t)$ is the space and time-dependent acoustic pressure in the pulsed ultrasound fields. Hereby (1) can be rewritten as

$\widehat{P}_{1}\left(k_{x}, k_{y}, z_{1}, f\right)=\widehat{P}_{0}\left(k_{x}, k_{y}, z_{0}, f\right) H_{p}^{f}\left(k_{x}, k_{y}, f\right)$,

where $H_{p}^{f}\left(k_{x}, k_{y}, f\right)$ is different from the monochromatic angular spectrum propagator $H_{p}\left(k_{x}, k_{y}\right)$ in (2), since the temporal frequency $f$ is a variable in the pulsed ultrasound fields. To satisfy that the acoustic wave propagates along $+z$ direction for both positive and negative frequency $f, H_{p}^{f}\left(k_{x}, k_{y}, f\right)$ has to be calculated by

$H_{p}^{f}\left(k_{x}, k_{y}, f\right)=\left\{\begin{array}{l}e^{-s g n(f) j\left(z_{1}-z_{0}\right) \sqrt{k^{2}-k_{x}^{2}-k_{y}^{2}}}, \text { when } k^{2}>k_{x}^{2}+k_{y}^{2}, \\ e^{-\left(z_{1}-z_{0}\right) \sqrt{k_{x}^{2}+k_{y}^{2}-k^{2}}}, \text { when } k^{2} \leqslant k_{x}^{2}+k_{y}^{2},\end{array}\right.$

where $\operatorname{sgn}(f)$ denotes the sign of the frequency $f$ ( 1 for positive $f$ and -1 for negative $f$ ). Finally, the exact equation of the pulsed angular spectrum approach is given by $p_{1}\left(x, y, z_{1}, t\right)=\mathcal{F}_{3 D}^{-1}\left\{\mathcal{F}_{3 D}\left\{p_{0}\left(x, y, z_{0}, t\right)\right\} H_{p}^{f}\left(k_{x}, k_{y}, f\right)\right\}$,

where $\mathcal{F}_{3 D}\{\}$ and $\mathcal{F}_{3 D}^{-1}\{\}$ represent three-dimensional (2D for $x-y$ plane and 1D for time) forward and inverse Fourier transforms. The acoustic source, $p_{0}\left(x, y, z_{0}, t\right)$ can either be simulated by Field II or measured in water by a hydrophone.

\subsection{Implementation equations}

For implementation reasons, the discrete forms of (6) and (7), based on the three-dimensional forward and inverse discrete Fourier transforms are used as

$$
\begin{aligned}
p_{d_{1}}\left(i_{x}, i_{y}, i_{t}\right)= & \frac{1}{N_{x} N_{y} N_{t}} \sum_{n_{y}=0}^{N_{y}-1} \sum_{n_{x}=0}^{N_{x}-1} \sum_{n_{t}=0}^{N_{t}-1}\left\{\widehat{P}_{d_{0}}\left(n_{x}, n_{y}, n_{t}\right) H_{d}^{f}\left(n_{x}, n_{y}, n_{t}\right)\right\} \\
& \times e^{2 \pi j\left(\frac{i_{t} n_{t}}{N_{t}}+\frac{i_{x} n_{x}}{N_{x}}+\frac{i_{y} n_{y}}{N_{y}}\right)},\left\{\begin{array}{l}
i_{t}=0, \ldots N_{t}-1, \\
i_{x}=0, \ldots N_{x}-1 \\
i_{y}=0, \ldots N_{y}-1,
\end{array}\right.
\end{aligned}
$$

where

$$
\begin{aligned}
\widehat{P}_{d_{0}}\left(n_{x}, n_{y}, n_{t}\right)= & \sum_{i_{y}=0}^{N_{y}-1} \sum_{i_{x}=0}^{N_{x}-1} \sum_{i_{t}=0}^{N_{t}-1} p_{d_{0}}\left(i_{x}, i_{y}, i_{t}\right) \\
& \times e^{-2 \pi j\left(\frac{n_{t} i_{t}}{N_{t}}+\frac{n_{x} i_{x}}{N_{x}}+\frac{n_{y} i_{y}}{N_{y}}\right)},\left\{\begin{array}{l}
n_{t}=0, \ldots N_{t}-1, \\
n_{x}=0, \ldots N_{x}-1, \\
n_{y}=0, \ldots N_{y}-1,
\end{array}\right.
\end{aligned}
$$

and

$$
H_{d}^{f}\left(n_{x}, n_{y}, n_{t}\right)=\left\{\begin{array}{l}
e^{-2 \pi j\left(z_{1}-z_{0}\right)} \sqrt{\left(\frac{f_{s} n_{t}}{c N_{t}}\right)^{2}-\left(\frac{n_{x}}{d x y N_{x}}\right)^{2}-\left(\frac{n_{y}}{d_{x y} N_{y}}\right)^{2}} \\
\text { when }\left(\frac{f_{s} n_{t}}{c N_{t}}\right)^{2}>\left(\frac{n_{x}}{d_{x y} N_{x}}\right)^{2}+\left(\frac{n_{y}}{d_{x y} N_{y}}\right)^{2} \\
e^{-2 \pi\left(z_{1}-z_{0}\right)} \sqrt{\left(\frac{n_{x}}{d_{x y} N_{x}}\right)^{2}+\left(\frac{n_{y}}{d_{x y} N_{y}}\right)^{2}-\left(\frac{f_{s} n_{t}}{c N_{t}}\right)^{2}} \\
\text { when }\left(\frac{f_{s} n_{t}}{c N_{t}}\right)^{2} \leqslant\left(\frac{n_{x}}{d_{x y} N_{x}}\right)^{2}+\left(\frac{n_{y}}{d_{x y} N_{y}}\right)^{2}
\end{array}\right.
$$

where $p_{d_{0}}\left(i_{x}, i_{y}, i_{t}\right)$ and $p_{d_{1}}\left(i_{x}, i_{y}, i_{t}\right)$ are the discrete forms of $p_{0}\left(x, y, z_{0}, t\right)$ and $p_{1}\left(x, y, z_{1}, t\right)$ in (7). $N_{x}, N_{y}$ and $N_{t}$ are the numbers of spatial and temporal discrete points. $f_{s}$ is the temporal sampling frequency and $d_{x y}$ is the spatial interval. By implementing (8)-(10), $p_{d_{1}}\left(i_{x}, i_{y}, i_{t}\right)$ at $z=z_{1}$ is obtained by the ASA based on the $p_{d_{0}}\left(i_{x}, i_{y}, i_{t}\right)$, which is at $z=z_{0}$ and calculated by Field II or measured in water.

\section{Simulation and Measurement setup}

In ASA simulations, a known acoustic plane is required as the virtual source of the ASA. In this paper, both simulated and measured sources are used to make the ASA simulations, which are compared to the results in the simulated plane, obtained by Field II and measurements, respectively. This section shows the setups of the transducer, Field II, and experimental instruments, and parameters used in the implementation of the ASA.

\subsection{Transducer}

A spherically focused ultrasonic piston transducer (Type: M309, from Panametrics Inc., USA) is used in the measurement and configured by Field II in the ASA for the simulated source. The transducer has a center frequency of $5 \mathrm{MHz}$, and its diameter is 0.5 inch corresponding to $12.7 \mathrm{~mm}$. The excitation for the transducer is a 2-cycle sine wave. The geometrical focus lies $62 \mathrm{~mm}$ away from the transducer surface. 


\subsection{Simulation setup and parameter study}

The simulated ASA source is calculated by the Field II program. The function "xdc_concave" is used to generate the emitted aperture for the transducer mentioned in the previous section. The

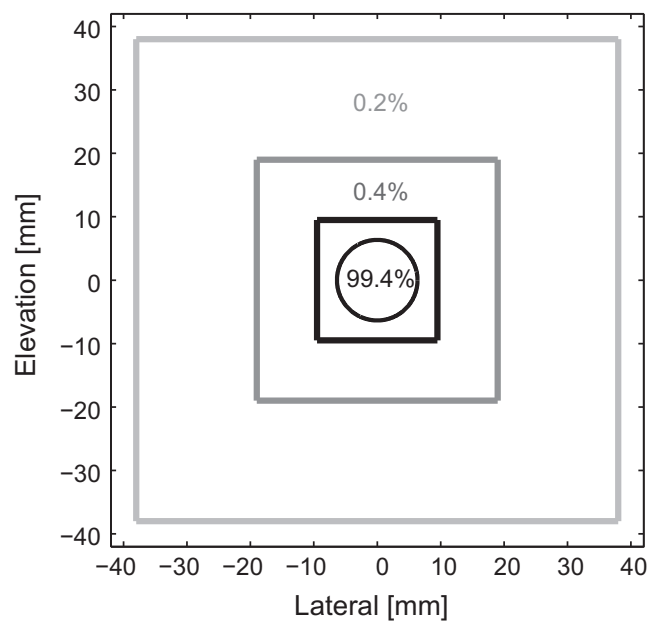

Fig. 1. The circle denotes the size of the transducer. The square is $12 \mathrm{~mm}$ from the transducer surface and denotes the size of the source plane for the ASA. If all the acoustic energy is in the largest gray square, then $99.4 \%$ of the energy is inside the smallest black square, $0.4 \%$ of the energy is between the middle and smallest squares and $0.2 \%$ of the energy is outside the middle square.

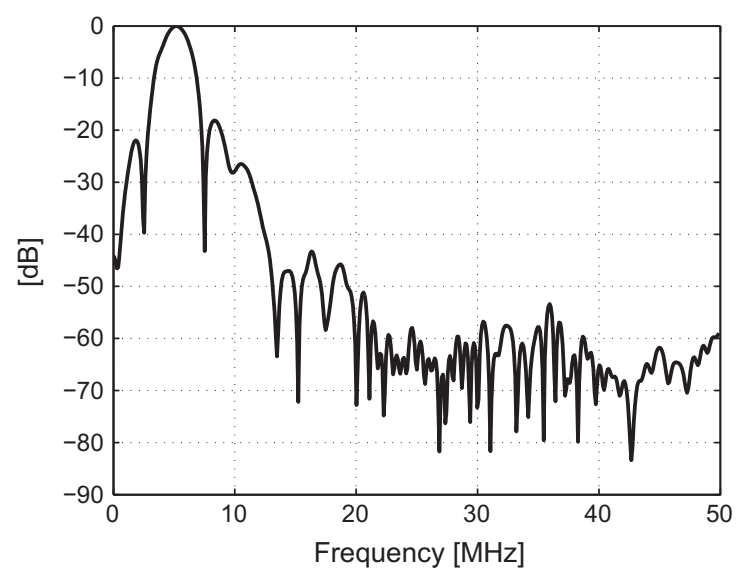

Fig. 2. Frequency spectrum of the emitted pulse generated by the transducer. propagating medium is water, and attenuation is not taken into consideration. The speed of sound in water is $1480 \mathrm{~m} / \mathrm{s}$ at normal temperature and ambient pressure. An acoustic plane, which is $12 \mathrm{~mm}$ from the transducer surface, is selected as the ASA source plane. On this plane, $p_{0}\left(x, y, z_{0}, t\right)$ in $(7)$ is calculated by Field II, where $z_{0}=12 \mathrm{~mm}$. To implement (8)-(10), several initial values are required. The length of the signals $N_{x}, N_{y}$ and $N_{t}$ in the spatial and time domains, which are related to the actual signals' length plus the zero-padding, and the temporal sampling frequency $f_{s}$ and spatial sampling interval $d_{x y}$ denoted in (10) should be initialized at first. A parameter study will be made in the simulation by choosing different initial values to search for an optimized ASA, which should give a relatively better accuracy with less computational complexity.

There are two constrains for the parameter selection. First, the source plane should contain all of the acoustic energy, so that it can be used as the virtual transmitting source to calculate the pressure in another parallel planes by the ASA. Secondly, the spatial and temporal sampling frequencies should be greater than twice the upper bandlimits of the signals in the spatial and temporal domains in accordance with Nyquist's sampling theory [21].

For the first constrain, the source planes with different sizes are simulated using Field II. Fig. 1 shows that $99.4 \%$ of the acoustic energy is inside the smallest square, if it is assumed that the largest square contains all of the energy. The acoustic energy is proportional to the root square (RS) values, which can be expressed as

RS value $=\sqrt{\sum_{i_{x}=0}^{N_{x}-1} \sum_{i_{y}=0}^{N_{y}-1} \sum_{i_{t}=0}^{N_{t}-1} p_{d}^{2}\left(i_{x}, i_{y}, i_{t}\right)}$,

where $p_{d}\left(i_{x}, i_{y}, i_{t}\right)$ is the discrete acoustic pressure at the source plane and is calculated by Field II. The spatial sampling interval equals half a wavelength $\left(\lambda=c / f_{0}=1480 /\left(5 \times 10^{6}\right)=0.296 \mathrm{~mm}\right)$, and the temporal sampling frequency is $100 \mathrm{MHz}$. Therefore, for example, $N_{x}$ are 128 and 512 for the smallest and largest squares corresponding to their sizes, respectively. The larger the square used as the source plane is, the more of the energy will be used. This will make the simulation more accurate, but also increase calculation time. The smallest square in Fig. 1 is used in the ASA simulations presented in the paper.

For the second constrain, the temporal sampling frequency is chosen according to the bandwidth of the transducer. Fig. 2 presents the frequency spectrum of the emitted pulse in the range from 0 to $50 \mathrm{MHz}$. The value of the spectrum is approximately below $-60 \mathrm{~dB}$ after $20 \mathrm{MHz}$. This reveals the upper bandlimit of the transducer is around $20 \mathrm{MHz}$. Thus, the temporal sampling frequency $f_{s}$ is set to $40 \mathrm{MHz}, 60 \mathrm{MHz}, 80 \mathrm{MHz}$ and $100 \mathrm{MHz}$ to study the accuracy of

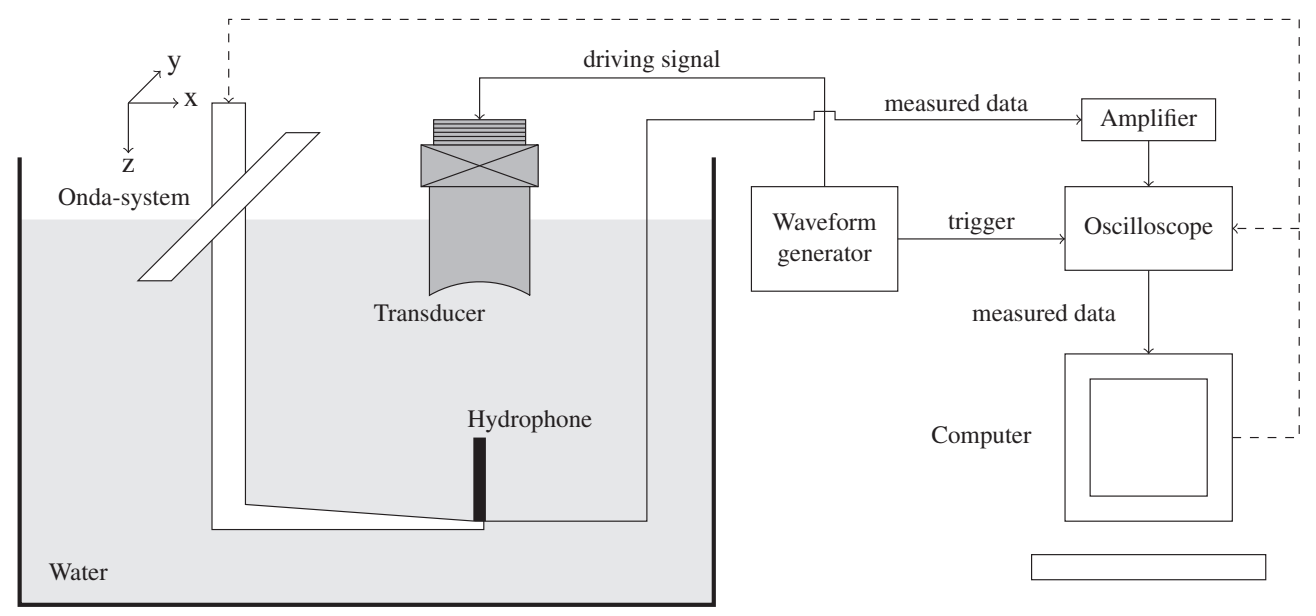

Fig. 3. Schematic diagram of the experimental setup. 


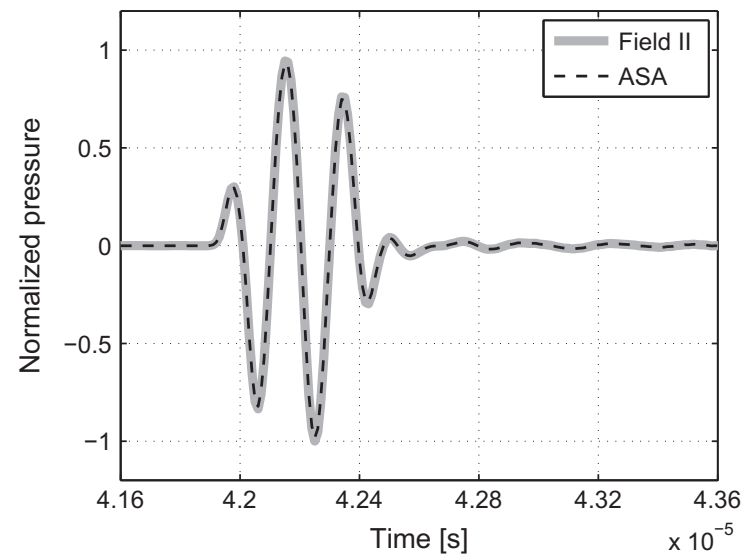

Fig. 4. Pulses at the focal point $z=62 \mathrm{~mm}$ away from the transducer surface. the simulation. Then four values $(\lambda / 2, \lambda, 4 \lambda / 3$ and $2 \lambda)$ are used for the spatial sampling interval, where $\lambda$ is the wavelength, denoted by $\lambda=c / f_{0}$ and $f_{0}=5 \mathrm{MHz}$ is the center frequency of the transducer.

Zero-padding is used to smooth the frequency spectrum in the temporal frequency domain and overcome the circular convolution effect in the spatial frequency domain. Zero-padding factors are 2 (meaning that the Fourier transform has twice the length of the input data), 4, 6 and 8 in the spatial domain. The number of sampling points (time-point) are 256, 512, 768, and 1024 for a pulse along time at a specific spatial position, where the signal at different locations approximately contains $110-150$ non-zero discrete values when $f_{s}=100 \mathrm{MHz}$.

\subsection{Measurement setup}

The experimental setup is shown in Fig. 3. The measurement is performed in water by an XYZ-Onda system - AIMS III (Acoustic

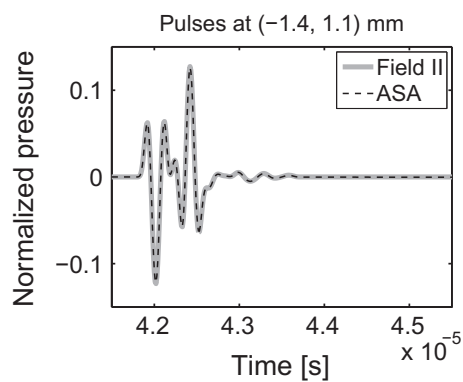

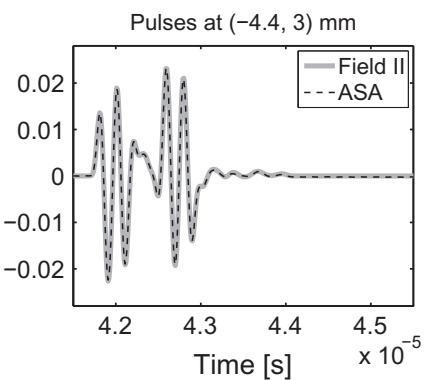



(a) Pulses at three positions of the focal plane $z=62 \mathrm{~mm}$

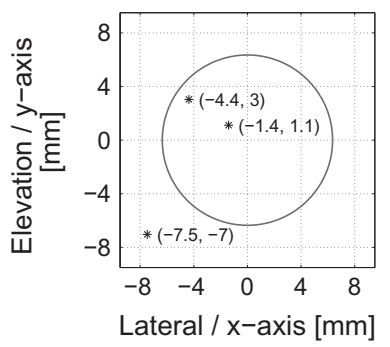

(b) Positions of those three points at focal plane


circle represents the size of the transducer.
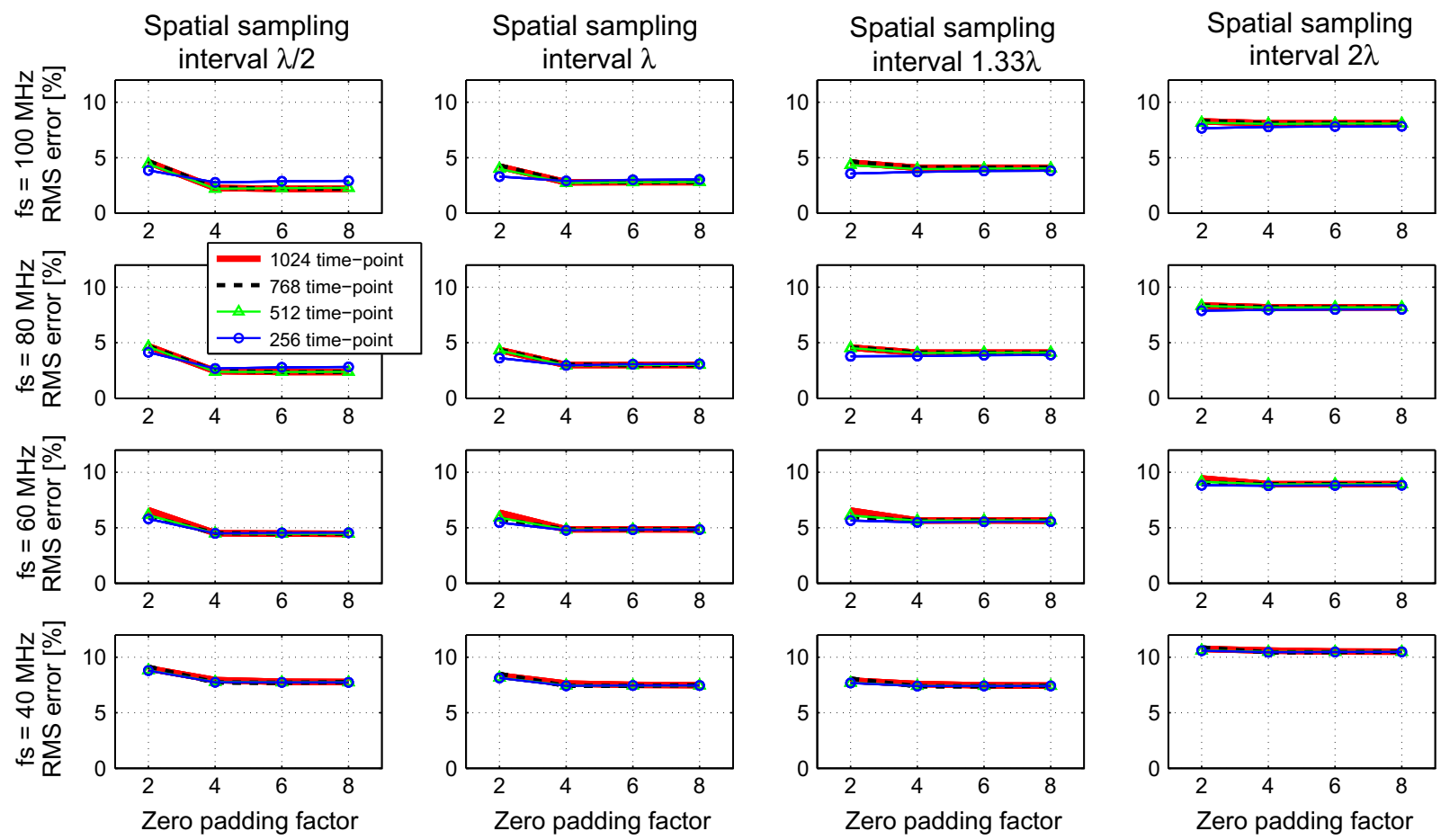

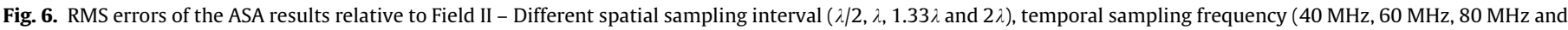
$100 \mathrm{MHz})$, spatial zero-padding factor $(2,4,6$ and 8$)$ and time-point $(256,512,768$ and 1024) are used in the ASA simulation to compare with Field II. 

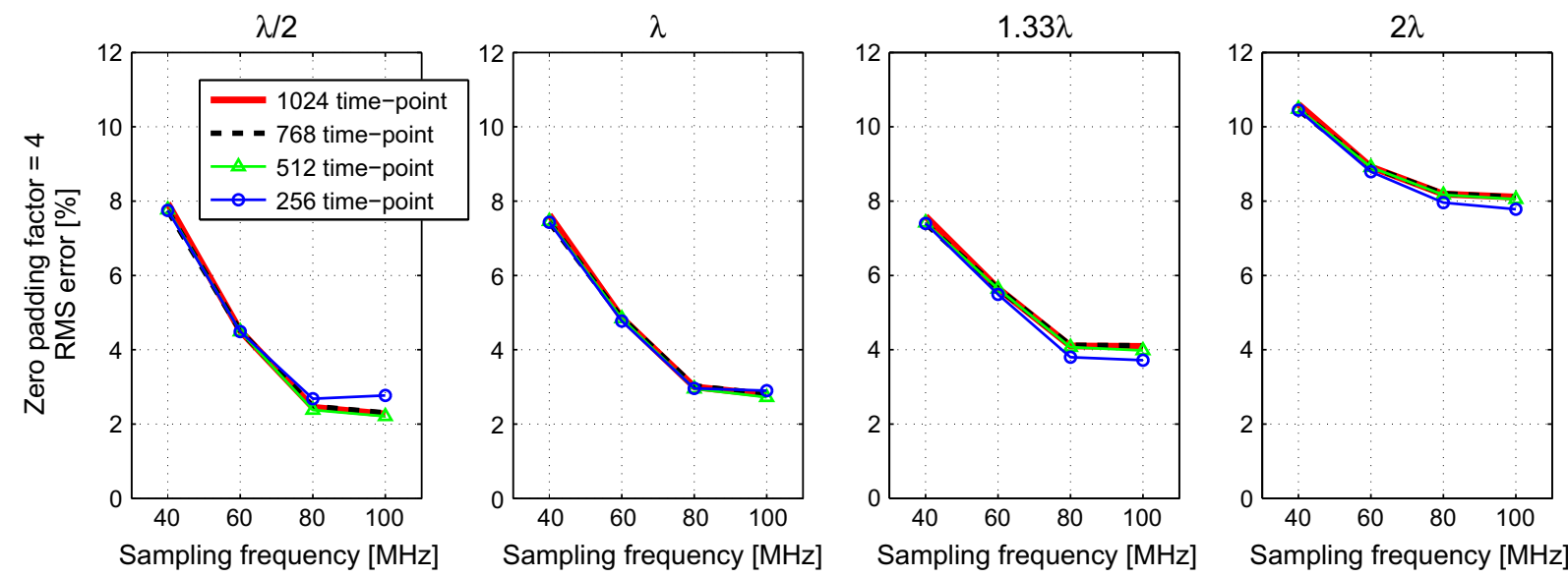


point.

Intensity Measurement System). The transducer is fixed, and a robust Onda hydrophone is displaced by the XYZ-Onda system to measure the pulse for all the points on the acoustic plane. The $\mathrm{XYZ}$-Onda system is controlled through a computer program Soniq 5.0. An Agilent waveform generator provides the driving signal for the transducer and a trigger pulse for an Agilent oscilloscope. The output voltage of the waveform generator for the transducer is 10 volts. When the transducer starts emissions, the oscilloscope will record the signals synchronously. The data obtained by the hydrophone after amplification will be recorded by the oscilloscope, which is controlled by computer programs as well. The data are stored in the computer memory for post-processing. Two planes at $32 \mathrm{~mm}$ and at $62 \mathrm{~mm}$ from the transducer surface are measured by the hydrophone.

\section{Results}

\subsection{ASA compared to Field II}

A comparison of pulses simulated by pulsed ASA and Field II at the focal point is shown in Fig. 4. To further validate it, three random points $(-1.4,1.1),(-4.4,3)$ and $(-7.5,-7) \mathrm{mm}$ at the focal plane are selected, and the comparisons are shown in Fig. 5a. Fig. 5b denotes the positions of these three points at the focal plane where $z=62 \mathrm{~mm}$ from the transducer surface. The circle shows the size of the transducer. The results for the ASA shown in Figs. 4 and 5 are calculated using 1024 time-point, $100 \mathrm{MHz}$ temporal sampling frequency and half wavelength $(\lambda / 2)$ spatial interval with a zero-padding factor of 8 .

To further investigate the accuracy and computational complexity of the ASA simulation, the root mean square (RMS) errors between the results obtained by the ASA and Field II as

RMS error $=\sqrt{\frac{\sum_{i_{x}=0}^{N_{x}-1} \sum_{i_{y}=0}^{N_{y}-1} \sum_{i_{t}=0}^{N_{t}-1}\left(p_{\text {asa }}\left(i_{x}, i_{y}, i_{t}\right)-p_{\text {ref }}\left(i_{x}, i_{y}, i_{t}\right)\right)^{2}}{\sum_{i_{x}=0}^{N_{x}-1} \sum_{i_{y}=0}^{N_{y}-1} \sum_{i_{t}=0}^{N_{t}-1} p_{\text {ref }}^{2}\left(i_{x}, i_{y}, i_{t}\right)}} \times 100 \%$,

are calculated with different parameters setting as described in Section 3.2. The RMS errors are shown in Fig. 6, which displays the results at different temporal and spatial sampling frequencies, and different time-point and zero-padding. After observing the trend of the RMS errors, it can be concluded:

1. The optimal (spatial) zero-padding factor is 4 from the observation of Fig. 6 as the error is not reduced much at all different settings, if the zero-padding factor is larger than 4 .
2. The optimal temporal sampling frequency is $80 \mathrm{MHz}$ according to Fig. 7, which shows the error trend as the increase of the sampling frequency with the optimal fixed zero-padding factor of 4.

3. Figs. 6-8 reveal that the different time-point gives trivial differences except for using 256 time-point. The blue circle solid curves in each figure are different from others - green angle solid, black dash and red solid lines.

4. The RMS error is increased as the spatial sampling interval is enlarged from $\lambda / 2$ to $2 \lambda$. However, the difference between using $\lambda / 2$ and $\lambda$ is relatively small as shown in Fig. 8.

The maximum RMS error is $10.9 \%$, while the zero-padding factor is 2 , the temporal sampling frequency is $40 \mathrm{MHz}$, and the spatial sampling interval is $2 \lambda$ with 768 time-point for each pulse. The RMS error with optimal parameters (zero-padding factor $=2$; temporal sampling frequency $=80 \mathrm{MHz}$; time-point $=512$; spatial sampling interval $=\lambda / 2$ ) is reduced to $2.4 \%$.

\subsection{ASA compared to measurements}

The measured and ASA simulated pulses at the focal point are shown in Fig. 9. The optimal parameters (zero-padding factor, temporal sampling frequency, time-point and spatial sampling inter-

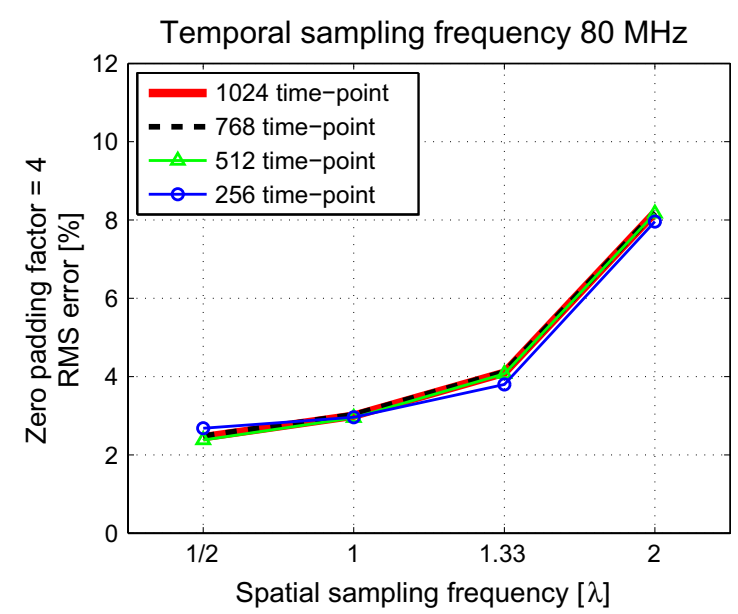

Fig. 8. RMS errors of the ASA results referred to Field II - a fixed zero-padding factor (4) and temporal sampling frequency $(80 \mathrm{MHz})$ are used with different time-point. The errors are along with spatial sampling frequency $(\lambda / 2, \lambda, 1.33 \lambda$ and $2 \lambda)$ as shown in the horizontal axis. 


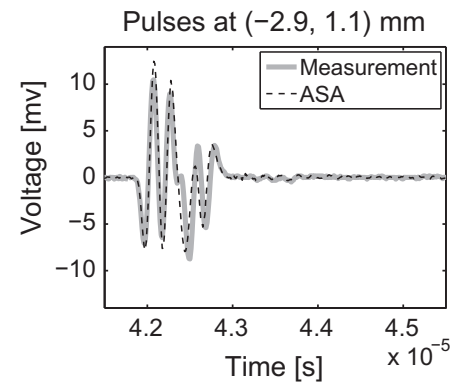

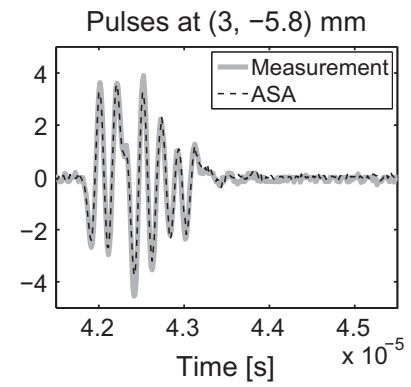

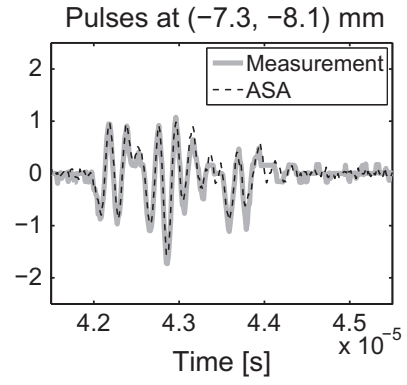

(a) Pulses at three positions of the focal plane $z=62 \mathrm{~mm}$

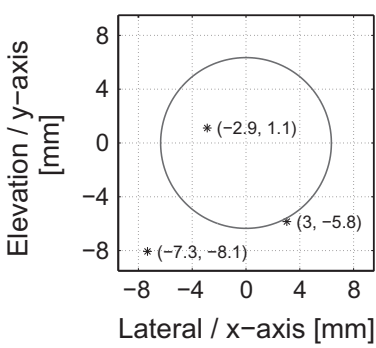

(b) Positions of those three points at focal plane

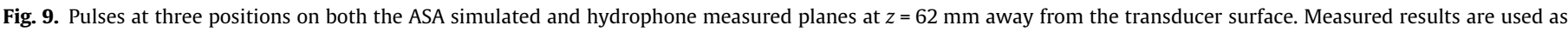
the ASA source plane. The circle represents the size of the transducer.

val) presented in the simulation are selected for the ASA with measured source. When the ASA calculates the pulse at the simulated plane, the accuracy of the sound speed becomes an issue, since the time line for the ASA calculated pulse is very dependent of the true sound speed in water for the measurement according to (7) as $k=2 \pi f / c$. Assume that the sound speed in water approximately equals $1480 \mathrm{~m} / \mathrm{s}$ according to the temperature during the measurements. However, it will be very difficult to know an exact value of the real sound speed in water during each measurement. Even a sound speed error of $0.5 \mathrm{~m} / \mathrm{s}$ can cause an obvious shift for the pulse calculated by the ASA compared to the measured pulse. To find the correct sound speed, the time lag $\Delta t$ between the pulse calculated by the ASA using the assumed sound speed $1480 \mathrm{~m} / \mathrm{s}$ and the measured pulse, can be expressed as

$\Delta t=\frac{z_{1}-z_{0}}{c_{a}}-\frac{z_{1}-z_{0}}{c_{r}}$,

where $z_{1}-z_{0}=30 \mathrm{~mm}$ is the distance between two measured planes. $c_{a}$ is the assumed sound speed $1480 \mathrm{~m} / \mathrm{s}$ used in the ASA simulation, and $c_{r}$ is the real sound speed during measurements and is the unknown variable in the equation. The time lag $\Delta t$ can be found by applying the cross-correlation function to these calculated and measured pulses. By solving (13), $c_{r}$ is obtained and reused in the ASA simulation. Thus, pulses between the ASA simulation and measurements are attained in phase as shown in Fig. 9. The RMS error between the ASA simulated and hydrophone measured pulses is $25.4 \%$ calculated by (12). The average sound speed is determined to be $1487.6 \mathrm{~m} / \mathrm{s}$ using (13). This can be applied to approximate the value of ambient sound speed under the



Fig. 10. The vertical axis presents the estimated true sound speed during the hydrophone measurement; The horizontal axis presents the initial used sound speed in the ASA simulation. linear acoustic propagation. To investigate the accuracy of this approach, different initial sound speeds are used in the ASA simulation. The corresponding estimated sound speeds are calculated using (13). The results are shown in Fig. 10, where the initially assumed sound speed varies from $1445 \mathrm{~m} / \mathrm{s}$ to $1540 \mathrm{~m} / \mathrm{s}$ in the ASA simulation. The calculated ambient sound speed in the measurement is in the range between $1487.45 \mathrm{~m} / \mathrm{s}$ and $1487.75 \mathrm{~m} / \mathrm{s}$ as shown in Fig. 10 and is only minimally influenced by the initially assumed sound speed.

\section{Conclusion}

An angular spectrum approach for simulating pulsed ultrasound fields is formulated and implemented in this paper. Investigations of different time-point, zero-padding factors, spatial sampling interval and temporal sampling frequency are made to find the optimal parameters for the ASA. The RMS error for the results obtained by the optimized ASA using a simulated source relative to Field II is $2.4 \%$. The ASA with a measured source has been implemented and simulations have been verified by measured results. The correct sound speed in the measurement is approximated using the formula presented in the study. This also proposes a potential approach for measuring the speed of the ambient acoustic wave propagation in water.

\section{Acknowledgements}

The authors thank our colleagues Ye Li and An Hoai Pham at the Center for Fast Ultrasound Imaging, Technical University of Denmark, for the help in the setup of experimental measurements. The project was supported by Grant 08-032480 from the Danish Agency for Science, Technology and Innovation, and BK Medical Aps, Denmark.

\section{References}

[1] P.R. Stepanishen, K.C. Benjamin, Forward and backward projection of acoustic fields using FFT methods, Journal of the Acoustical Society of America 71 (4) (1982) 803-812.

[2] D.P. Orofino, P.C. Pedersen, Efficient angular spectrum decomposition of acoustic sources. Part I: theory, IEEE Transactions on Ultrasonics, Ferroelectrics, and Frequency Control 40 (3) (1993) 238-249.

[3] D.P. Orofino, P.C. Pedersen, Efficient angular spectrum decomposition of acoustic sources. Part II: results, IEEE Transactions on Ultrasonics, Ferroelectrics, and Frequency Control 40 (3) (1993) 250-257.

[4] J.W. Goodman, Introduction to Fourier Optics, McGraw-Hill, New York, USA, 1968.

[5] J.D. Gaskill, Linear Systems, Fourier Transforms, and Optics (Wiley Series in Pure and Applied Optics), Wiley-Interscience, New York, USA, 1978.

[6] X. Zeng, R.J. McGough, Evaluation of the angular spectrum approach for simulations of near-field pressures, Journal of the Acoustical Society of America 123 (1) (2007) 68-76. 
[7] X. Zeng, R.J. McGough, Optimal simulations of ultrasonic fields produced by large thermal therapy arrays using the angular spectrum approach, Journal of the Acoustical Society of America 125 (5) (2009) 2967-2977.

[8] X. Zeng, R.J. McGough, Evaluation of angular spectrum approach for simulations of spherically focused ultrasound phased arrays, in: Proceedings of the IEEE Electro/Information Technology, 2006, pp. 66-71.

[9] P. Wu, T. Stepinski, Extension of the angular spectrum approach to curved radiators, Journal of the Acoustical Society of America 105 (5) (1999) 2618-2627.

[10] P. Alais, P.Y. Hennion, A Fourier theory of the nonlinear interaction of acoustical beams in absorbing fluid. The special case of parametric emission, Acustica 43 (1979) 1-11.

[11] B.J. Landsberger, M.F. Hamilton, Second-harmonic generation in sound beams reflected from, and transmitted through, immersed elastic solids, Journal of the Acoustical Society of America 109 (2) (2001) 488-500.

[12] Y. Xiang, M.F. Hamilton, Angular spectrum decomposition analysis of second harmonic ultrasound propagation and its relation to tissue harmonic imaging, in: Proceedings of the 4th International Workshop on Ultrasonic and Advanced Methods for Nondestructive Testing and Material Characterization, vol. UmassD-NDT, 2006, pp. 11-24.

[13] Y. Du, H. Jensen, J.A. Jensen, Comparison of simulated and measured non-linear ultrasound fields, in: Proceedings of the SPIE Medical Imaging, vol. 79680P 2011, pp. 1-10.
[14] A.J. Healey, S. Leeman, J.P. Weight, Space-time imaging of transient ultrasound fields, International Journal of Imaging Systems and Technology 8 (1997) 4551

[15] S. Leeman, A.J. Healey, Field analysis with a new field simulation and propagation technique, Acoustical Imaging 25 (2000) 41-50.

[16] P. Wu, R. Kazys, T. Stepinski, Calculation of transient fields in immersed solids radiated by linear focuing arrays, in: Proceedings of the IEEE Ultrasonics Symposium, 1995, pp. 993-997.

[17] R.J. Zemp, J. Tavakkoli, R.S.C. Cobbold, Modeling of nonlinear ultrasound propagation in tissue from array transducers, Journal of the Acoustical Society of America 113 (1) (2003) 139-152.

[18] T. Varslot, G. Taraldsen, Computer simulation of forward wave propagation in soft tissue, IEEE Transactions on Ultrasonics, Ferroelectrics, and Frequency Control 52 (9) (2005) 1473-1482.

[19] J.A. Jensen, N.B. Svendsen, Calculation of pressure fields from arbitrarily shaped, apodized, and excited ultrasound transducers, IEEE Transactions on Ultrasonics, Ferroelectrics, and Frequency Control 39 (2) (1992) 262-267.

[20] J.A. Jensen, Field: A program for simulating ultrasound systems, in: Medical and Biological Engineering and Computing, vol. 34, 1996, pp. 351-353.

[21] C. Shannon, Communication in the presence of noise, in: Proceedings of the IEEE, vol. 37, 1949, pp. 10-21. 